\section{Saúde bucal dos índios Guaraní no Estado do Rio de Janeiro, Brasil}

\author{
Oral health of Guaraní Indians in the State of \\ Rio de Janeiro, Brazil
}

${ }^{1}$ Equipe Multidisciplinar de Saúde Indígena, Fundação Nacional de Saúde, Rio de Janeiro, Brasil.

2 Fundação de Saúde de Angra dos Reis, Angra dos Reis, Brasil.

3 Escola Nacional de Saúde Pública Sergio Arouca Fundação Oswaldo Cruz, Rio de Janeiro, Brasil. ${ }^{4}$ Museu Nacional,

Universidade Federal do Rio de Janeiro, Rio de Janeiro,

Brasil.

Correspondência R. V. Santos

Departamento de Endemias Samuel Pessoa, Escola Nacional de Saúde Pública Sergio Arouca, Fundação Oswaldo Cruz.

Rua Leopoldo Bulhões 1480,

Rio de Janeiro, $R J$

21041-210, Brasil.

santos@ensp.fiocruz.br

\section{Abstract}

The aim of the present study was to describe the oral health conditions of Guarani Indians in the State of Rio de Janeiro, Brazil. A cross-sectional oral health survey was conducted in 508 subjects (90.7\% of the Guarani population) using World Health Organization criteria. At 5 years of age, $38.5 \%$ of children were caries-free. DMF-T was 1.7 at 12 years. $54.2 \%$ of subjects in the 35-44year group and $12 \%$ in the 65-74-year group had at least 20 teeth. Numbers of teeth for the 35-44 and 65-74 age groups were 18.5 and 8.5, respectively. While caries index did not differ by gender for deciduous teeth, DMF-T and tooth loss was higher in women. Nearly 60\% of the population showed no periodontal illness. Gingival bleeding and calculus were the most severe periodontal conditions. Gingival bleeding and calculus were more common in men and excluded sextants more common in women. Oral health promotion strategies are needed for this ethnic group and should consider the epidemiological profile according to age and gender.

Dental Caries; Oral Health; South American Indians
Pedro Alves Filho 1,2

Ricardo Ventura Santos 3,4

Mario Viana Vettore 3

\section{Introdução}

A avaliação da situação de saúde bucal de comunidades é realizada, essencialmente, pelo estudo da cárie dentária e das doenças periodontais. Por ser mais freqüente, em indígenas e não indígenas, a cárie tem sido mais investigada do ponto de vista epidemiológico, com a finalidade de construir conhecimentos acerca de sua determinação, distribuição e prevalência, assim como elaborar estratégias preventivas 1,2,3.

Vivem no Brasil mais de 200 etnias indígenas, distribuídas em todo o território nacional. Os estudos sobre saúde bucal indígena, ainda em pequeno número no país frente à sócio-diversidade existente, além de concentrados na região amazônica, apontam para uma grande heterogeneidade epidemiológica 4,5. Tal como em outras populações, entre os povos indígenas, e no caso da cárie em particular, os padrões patognomônicos guardam uma estreita relação com determinantes culturais, comportamentais e biológicos (dieta, exposição ao flúor, entre outros) 5 . Os estudos de caso disponíveis sugerem uma tendência de aumento da prevalência da cárie nas populações indígenas 6,7,8,9,10,11. De maneira geral, esse aumento é atribuído ao impacto das mudanças na alimentação, associadas às modificações sócio-econômicas, ambientais e à falta de programas preventivos. Além de contribuir para o conhecimento acerca da multicausalidade da cárie, as investigações sobre saúde bucal indíge- 
na podem gerar informações relevantes para o planejamento e gestão dos serviços de saúde.

Para os povos indígenas localizados no SulSudeste do Brasil, os poucos estudos epidemiológicos sobre saúde bucal têm se concentrado em comunidades da etnia Guaraní 12,13. Tal como em outros estudos conduzidos em outras regiões do país, os resultados apontam para elevadas prevalências de cárie.

Este estudo teve como objetivo analisar a situação de saúde bucal dos índios Guaraní do Estado do Rio de Janeiro, atendidos na área de abrangência do Pólo-Base de Angra dos Reis (municípios de Angra dos Reis e Paraty). Além de comparar o perfil epidemiológico bucal entre sexos e faixas etárias, os resultados para os Guaraní são discutidos à luz de investigações conduzidas em outras etnias indígenas no Brasil, o que permite tecer considerações sobre a atenção à saúde que vem sendo provida a esses povos.

\section{População e métodos}

\section{Os Guaraní}

Os Guaraní são povos Tupi cuja população no Brasil é de aproximadamente 45 mil pessoas, o que representa em torno de $10 \%$ da população indígena no país. Os Guaraní são usualmente agrupados em três subgrupos: os Nhandéva, que constituem a maior população da etnia em território brasileiro; os Mbyá, que integram a maioria das aldeias na Região Sudeste; e os Kaiwá, cuja presença é maior no Mato Grosso do Sul. Os Guaraní que atualmente habitam aldeamentos no litoral de São Paulo, Rio de Janeiro (em particular nos municípios de Angra dos Reis e Paraty) e Espírito Santos (na região de Aracruz) fazem parte dos subgrupos Nhandéva e Mbyá 14 .

\section{Local da pesquisa}

A pesquisa foi realizada no Pólo-Base de Angra dos Reis, que integra o Distrito Sanitário Especial Indígena (DSEI) Litoral Sul e é um dos 34 DSEIs existentes no país. Os Guaraní do DSEI Litoral Sul totalizavam 5.216 indivíduos em 2006. Desses, 617 viviam em cinco aldeias (Sapukai, Rio Pequeno, Paratimirim, Araponga e Mamanguá) atendidas pelo Pólo-Base de Angra dos Reis.

A maior aldeia é a de Sapukai, que se localiza no Município de Angra dos Reis (latitudes

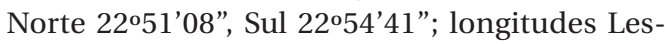
te $44^{\circ} 20^{\prime} 47^{\prime \prime}$, Oeste $44^{\circ} 24^{\prime} 41^{\prime \prime}$ ) 15 . Nessa aldeia vivem cerca de 360 Guaraní, distribuídos em 50 domicílios. Todas as demais aldeias estão situadas no Município de Paraty. Paratimirim (lati-

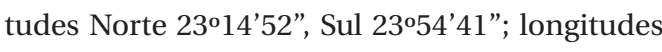

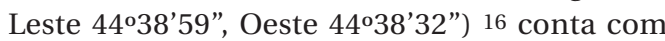
uma população de 180 pessoas, divididas em 32 domicílios. Araponga (latitudes Norte 2318'14", Sul 2319'04"; longitudes Leste $44^{\circ} 45^{\prime} 45^{\prime}$, Oeste $\left.44^{\circ} 47^{\prime} 11^{\prime \prime}\right)$ apresenta uma população pequena, de apenas vinte indivíduos, que vivem em seis domicílios 17. Rio Pequeno conta com 18 pessoas, em quatro domicílios. Finalmente, em Mamanguá residem 23 pessoas, que vivem em cinco domicílios. Não há informações sobre coordenadas geográficas para essas duas últimas, pois as mesmas ainda não foram demarcadas.

$\mathrm{O}$ acesso a todas as comunidades, com exceção de Mamanguá (onde só se chega por mar), pode ser feito por terra. Atualmente os Guaraní que vivem na região de Angra dos Reis e Paraty têm uma economia baseada em roças de subsistência e na venda de artesanato. Há também o influxo de recursos financeiros por meio de aposentadorias. A dieta da população inclui uma combinação de alimentos cultivados e industrializados. Apesar de viverem próximos do litoral, a pesca não é a principal atividade econômica da população.

\section{Metodologia}

Foi conduzido um estudo epidemiológico transversal do tipo inquérito. O estudo contemplou a análise das condições de saúde bucal dos índios Guaraní que residiam em todas as aldeias adscritas ao Pólo-Base de Angra dos Reis. Dados primários relativos às variáveis demográficas (idade e sexo), bem como sobre às condições de saúde bucal com base em índices preconizados pela Organização Mundial da Saúde (OMS), foram obtidos entre dezembro de 2006 e abril de 2007.

Participaram do estudo indivíduos com 18 meses ou mais de idade que eram residentes permanentes nas aldeias do Pólo-Base de Angra dos Reis e que possuíam inscrição no cadastro da família indígena (CAFI) do Sistema de Informação da Atenção à Saúde Indígena (SIASI), mantido pela Fundação Nacional de Saúde (FUNASA). As idades foram obtidas a partir de consulta ao CAFI. Foram visitados 97 domicílios e examinados todos os moradores presentes. A coleta de dados aconteceu após a anuência dos indivíduos e das lideranças indígenas.

Os exames clínicos foram realizados sob condições similares, com os pacientes sentados, sob luz natural e com a utilização de materiais e instrumentais clínicos esterilizados (espelho bucal plano, sonda periodontal milimetrada de Williams e gaze). Um único odontólogo, previamente calibrado, realizou os exames 18 . 
O coeficiente kappa foi utilizado para testar a concordância intra-examinador. Um total de 16 indivíduos de várias faixas etárias foram examinados e, posteriormente, reexaminados. Foi obtido um nível de concordância de 0,98 para o CPOD/ceod e 0,91 para o CPI (Índice Periodontal Comunitário) 18.

A avaliação das condições de saúde bucal incluiu análise da presença de cárie dentária, o que foi avaliado pelo índice ceo-d para dentes decíduos e pelo CPOD para dentes permanentes, assim como por seus componentes (dentes cariados, perdidos e obturados). O cálculo da composição percentual para os componentes do CPOD foi realizado utilizando-se os quocientes das médias dos elementos cariados, perdidos e obturados em relação à média total do índice. Foram também empregados os seguintes índices periodontais: Índice de Alterações Gengivais para os indivíduos com 5 anos de idade, e o CPI para aqueles com 12 ou mais anos.

O detalhamento dessas medidas pode ser encontrado nos manuais técnicos para levantamentos epidemiológicos em saúde bucal publicados pelo Ministério da Saúde 19. Esses manuais seguem diretrizes da OMS 20.

Foram realizadas análises das freqüências absolutas e relativas dos índices ceod, CPOD, CPI e de seus respectivos componentes. Analisou-se também a distribuição do Índice de Alterações Gengivais.

Os resultados são apresentados segundo estratos de idade e sexo. A normalidade da distribuição dos índices ceod e CPOD e de seus componentes foi avaliada pelo teste de Kolmogorov-Smirnov, que não evidenciou distribuição normal. Por essa razão, as médias desses índices foram comparadas utilizando-se o teste de Mann-Whitney. As proporções de indivíduos com alterações gengivais e segundo o CPI foram comparadas usando-se o teste qui-quadrado. Foram realizadas análises ajustadas para idade nas comparações de médias do CPOD e do número de dentes entre sexos empregando-se modelo linear generalizado.

O presente estudo foi aprovado pelo Comitê de Ética em Pesquisa da Escola Nacional de Saúde Pública Sergio Arouca, Fundação Oswaldo Cruz (parecer no. 114/06) e pela Comissão Nacional de Ética em Pesquisa (registro no ${ }^{\circ}$ 13663).

\section{Resultados}

Do total de 560 indivíduos elegíveis, 508 (90,7\%) foram examinados. A composição por sexo e idade da amostra investigada foi bastante próxima daquela da população total das comunida- des. Assim, a amostra incluiu 51,4\% de homens e $48,6 \%$ de mulheres, enquanto na população as porcentagens eram de $52,3 \%$ e $47,7 \%$, respectivamente. Quanto às faixas etárias, $40,7 \%$ tinham entre 18 meses e 10 anos (37,2\% da população), $22,2 \%$ de 12 a 19 anos (20,3\% da população) e $37,1 \%$ acima de 20 anos (42,5\% da população).

As médias de idade dos indivíduos dos sexos masculino e feminino foram, respectivamente, de 18,7 (desvio-padrão - DP = 19,6) e 21,1 (DP = $21,2)$ ( $p=0,045)$. Não foram detectadas diferenças estatisticamente significantes nas médias de idade nas várias faixas etárias segundo sexo $(\mathrm{p}>$ $0,05)$.

A análise do indicador ceod para crianças entre 18 e 36 meses evidenciou um valor de 0,6 (Tabela 1). O ceod foi de 2,6 aos 5 anos e alcançou o maior valor aos 8 anos de idade $(5,1)$. Entre 18 meses e 12 anos, o componente cariado foi o mais freqüente, correspondendo a 92,3\% do índice aos 5 anos e a $100 \%$ aos 12 . O componente obturado foi observado apenas em poucas faixas etárias (de 5 a 9 anos), alcançando o maior valor aos 9 anos (14,8\%). Somente 38,5\% das crianças de 5 anos estavam livres de cárie (dado não apresentado).

Na Tabela 2 são apresentadas as médias do ceo-d segundo sexo. Não há um padrão consistente de médias menos ou mais elevadas entre os sexos segundo estratos etários. Não foram detectadas diferenças estatisticamente significativas para o ceo-d entre sexos.

O CPOD médio da população foi de 6,3 (DP = 9,2 ) (Tabela 3). A presença de dentes permanentes atacados por cárie foi observada a partir dos 6 anos. A média aos 12 anos foi de 1,7, alcançando 9,0 entre 25 e 34 anos e 24,8 de 65 a 74 anos. Entre os 7 e 19 anos, as médias de elementos cariados se mantiveram mais elevadas que as de elementos obturados. Entre 20 e 24 anos a média do componente obturado esteve ligeiramente acima do perdido. Já a partir dos 25 anos as médias de dentes perdidos foram consistentemente superiores àquelas dos demais componentes do índice. $\mathrm{O}$ número de dentes experimentou redução com o aumento da idade, caindo de uma média de 25,4 na faixa etária de 20-24 anos para 4,2 nas pessoas com 75 anos ou mais.

Quanto aos componentes do CPOD, o percentual de elementos cariados superou os demais componentes do índice nas idades de 7 a 10 anos (80\%) e aos 12 anos (52,9\%) (Tabela 3). Também atingiu valor expressivo entre 15 e 19 anos (46,4\%). O componente obturado representou $20 \%$ ou mais do índice nas faixas etárias de 6, 7 a 10, 15 a 19, 20 a 24 e 25 a 34 anos. Dentre os adultos, o componente obturado atingiu seu maior valor entre os 20 e 24 anos $(37,7 \%)$. A partir 
Tabela 1

Médias de dentes decíduos hígidos, cariados, obturados com cárie, obturados e perdidos, valor do ceod e distribuição percentual de seus componentes, de acordo com idades e faixas etárias selecionadas, em indivíduos Guaraní. Rio de Janeiro, Brasil, 2007.

\begin{tabular}{|c|c|c|c|c|c|c|c|c|c|c|c|}
\hline \multirow[t]{2}{*}{ Idade } & \multirow[t]{2}{*}{$\mathbf{N}$} & \multirow[t]{2}{*}{ Hígidos } & \multicolumn{2}{|c|}{ Cariados } & \multicolumn{2}{|c|}{ Obturados com cáries } & \multicolumn{2}{|c|}{ Obturados } & \multicolumn{2}{|c|}{ Perdidos } & \multirow[t]{2}{*}{$\operatorname{ceod}( \pm D P)$} \\
\hline & & & Média & $\%$ & Média & $\%$ & Média & $\%$ & Média & $\%$ & \\
\hline 18-36 meses & 64 & 17,9 & 0,6 & 100,0 & 0 & - & 0 & - & 0 & - & $0,6 \pm 2,4$ \\
\hline 4 anos & 14 & 17,5 & 2,4 & 96,0 & 0,1 & 4,0 & 0 & - & 0 & - & $2,5 \pm 3,8$ \\
\hline 5 anos & 39 & 16,6 & 2,4 & 92,3 & 0 & - & 0,2 & 7,7 & 0 & - & $2,6 \pm 3,7$ \\
\hline 6 anos & 15 & 13,7 & 3,9 & 88,6 & 0,1 & 2,4 & 0,2 & 4,5 & 0,2 & 4,5 & $4,4 \pm 3,6$ \\
\hline 7 anos & 19 & 11,9 & 4,1 & 74,5 & 0 & - & 0,5 & 5,5 & 0 & - & $4,6 \pm 3,4$ \\
\hline 8 anos & 18 & 7,3 & 4,8 & 94,1 & 0 & - & 0,3 & 5,9 & 0 & - & $5,1 \pm 3,1$ \\
\hline 9 anos & 20 & 7,7 & 2,0 & 74,0 & 0 & - & 0,4 & 14,8 & 0,3 & 11,2 & $2,7 \pm 2,6$ \\
\hline 10 anos & 17 & 4,8 & 1,3 & 92,8 & 0 & - & 0 & - & 0,1 & 7,2 & $1,4 \pm 1,6$ \\
\hline 12 anos & 40 & 0 & 0,1 & 100,0 & 0 & - & 0 & - & 0 & - & $0,1 \pm 0,6$ \\
\hline Total & 246 & 10,4 & 1,8 & 90,0 & 0,01 & 0,5 & 0,2 & 10,0 & 0,02 & 1,0 & $2,0 \pm 3,0$ \\
\hline
\end{tabular}

Tabela 2

Comparação de médias do índice ceo-d entre sexos, de acordo com idades e faixas etárias selecionadas, em indivíduos Guaraní. Rio de Janeiro, Brasil, 2007.

\begin{tabular}{lccc}
\hline Idade & Masculino & ceod & Valor \\
& $0,79 \pm 2,46$ & Feminino & * \\
\hline 18-36 meses & $1,33 \pm 1,86$ & $0,48 \pm 2,20$ & 0,534 \\
4 anos & $2,52 \pm 2,77$ & $3,38 \pm 4,72$ & 0,892 \\
5 anos & $2,67 \pm 3,88$ & $2,71 \pm 2,76$ & 0,761 \\
6 anos & $4,00 \pm 3,29$ & $5,56 \pm 3,01$ & 0,095 \\
12 anos & $0,22 \pm 0,94$ & $2,63 \pm 2,74$ & 0,073 \\
Total & $2,13 \pm 0,05$ & $0,00 \pm 0,00$ & 0,269 \\
\hline
\end{tabular}

* Valor de p a partir de teste de Mann-Whitney. manteve-se estatisticamente significativa mesmo após ajuste para a idade $(\mathrm{p}<0,05)$.

As proporções de pessoas com 20 ou mais dentes foram de $87,8 \%$ na faixa etária $25-34$ anos, $54,2 \%$ para $35-44,0 \%$ para $45-64,12 \%$ para $65-74$ anos e 10,5\% para 75 anos ou mais. A redução do número de pessoas com 20 ou mais dentes foi estatisticamente significativa como o aumento da idade $(\mathrm{p}<0,001)$.

Observou-se uma freqüência de alterações gengivais em crianças de cinco anos de idade de $12 \%$ para meninos e de $7,1 \%$ para meninas, não sendo esta diferença estatisticamente significativa $(\mathrm{p}=0,632)$.

Na Tabela 5 são apresentados os resultados relativos ao CPI segundo sexos e estratos etários. Observou-se que 59,6\% da amostra apresentavam todos os sextantes com saúde periodontal A presença de cálculo dental, como condição periodontal mais grave, foi detectada em $20,9 \%$, seguida de sangramento gengival $(11,3 \%)$ e bolsas de $4-5 \mathrm{~mm}(0,7 \%)$. Não foram detectados casos de pessoas com bolsas periodontais profundas (> 5mm). Um conjunto de 7,6\% de indivíduos não foi examinado pois os sextantes não apresentavam dentes.

Foi detectada diferença estatisticamente significativa para o CPI segundo sexo $(p=0,002)$. Em geral, sangramento, cálculo e bolsas $4-5 \mathrm{~mm}$ foram mais freqüentes em indivíduos do sexo masculino, enquanto sextantes sadios e excluídos foram mais comuns em mulheres. Análises segundo estratos etários revelaram que, aos 12 anos, $10 \%$ apresentavam cálculo dentário como condição periodontal mais grave. Nos indivíduos de 15 a 19 anos as freqüências de sangramento e 
Média de dentes permanentes hígidos, cariados, obturados com cárie, obturados e perdidos, valor do CPOD, média do número de dentes presentes e distribuição percentual dos componentes do CPOD, de acordo com idades e faixas etárias selecionadas, de indivíduos Guaraní. Rio de Janeiro, Brasil, 2007.

\begin{tabular}{|c|c|c|c|c|c|c|c|c|c|c|c|c|}
\hline \multirow[t]{2}{*}{ Idade (anos) } & \multirow[t]{2}{*}{$\mathbf{N}$} & \multirow[t]{2}{*}{ Hígidos } & \multicolumn{2}{|c|}{ Cariados } & \multicolumn{2}{|c|}{ Obturados com cáries } & \multicolumn{2}{|c|}{ Obturados } & \multicolumn{2}{|c|}{ Perdidos } & \multirow{2}{*}{$\begin{array}{l}\text { CPOD } \\
( \pm D P)\end{array}$} & \multirow{2}{*}{$\begin{array}{l}\text { Número } \\
\text { de dentes }\end{array}$} \\
\hline & & & Média & $\%$ & Média & $\%$ & Média & $\%$ & Média & $\%$ & & \\
\hline 5 & 39 & 1,5 & 0 & - & 0 & - & 0 & - & 0 & - & $0,0 \pm 0,0$ & - \\
\hline 6 & 15 & 1,9 & 0,1 & 50,0 & 0 & - & 0,1 & 50,0 & 0 & - & $0,2 \pm 0,7$ & - \\
\hline $7-10$ & 74 & 11,8 & 0,8 & 80,0 & 0 & - & 0,2 & 20,0 & 0 & - & $1,0 \pm 1,2$ & - \\
\hline 12 & 40 & 24,0 & 0,9 & 52,9 & 0 & - & 0,6 & 35,3 & 0,2 & 11,8 & $1,7 \pm 1,9$ & - \\
\hline $15-19$ & 73 & 25,1 & 1,3 & 46,4 & 0,1 & 3,7 & 0,9 & 32,1 & 0,5 & 17,8 & $2,8 \pm 2,1$ & - \\
\hline $20-24$ & 29 & 23,8 & 1,5 & 28,3 & 0,1 & 1,9 & 2,0 & 37,7 & 1,7 & 32,1 & $5,3 \pm 3,0$ & 25,4 \\
\hline $25-34$ & 49 & 22,0 & 1,6 & 17,7 & 0,1 & 1,2 & 2,2 & 24,4 & 5,1 & 56,7 & $9,0 \pm 5,2$ & 23,7 \\
\hline $35-44$ & 48 & 17,3 & 1,2 & 8,6 & 0,1 & 0,8 & 2,0 & 14,4 & 10,6 & 76,2 & $13,9 \pm 7,9$ & 18,5 \\
\hline $45-64$ & 18 & 8,9 & 1,2 & 5,2 & 0,1 & 0,4 & 0,3 & 1,3 & 21,5 & 93,1 & $23,1 \pm 4,5$ & 10,2 \\
\hline $65-74$ & 25 & 7,2 & 1,3 & 5,2 & 0 & - & 0,1 & 0,5 & 23,4 & 94,3 & $24,8 \pm 7,0$ & 8,5 \\
\hline$\geq 75$ & 19 & 3,3 & 0,9 & 3,1 & 0 & - & 0,2 & 0,7 & 27,6 & 96,2 & $28,7 \pm 5,6$ & 4,2 \\
\hline Total & 508 & 13,4 & 0,9 & 14,3 & 0 & - & 0,8 & 12,7 & 4,6 & 73,0 & $6,3 \pm 9,2$ & 20,6 \\
\hline
\end{tabular}

Tabela 4

Comparação de médias do índice CPOD e número de dentes entre sexos, de acordo com idades e faixas etárias selecionadas, entre indivíduos Guaraní. Rio de Janeiro, Brasil, 2007.

\begin{tabular}{|c|c|c|c|c|c|c|}
\hline \multirow[t]{2}{*}{ Idade (anos) } & \multicolumn{3}{|c|}{ CPOD } & \multicolumn{3}{|c|}{ Número de dentes } \\
\hline & Masculino & Feminino & Valor de $p$ * & Masculino & Feminino & Valor de $p$ * \\
\hline 5 & $0,0 \pm 0,0$ & $0,0 \pm 0,0$ & 1,000 & - & - & - \\
\hline 6 & $0,3 \pm 0,8$ & $0,2 \pm 0,7$ & 0,7625 & - & - & - \\
\hline $7-10$ & $0,8 \pm 1,2$ & $0,7 \pm 1,1$ & 0,792 & - & - & - \\
\hline 12 & $1,8 \pm 2,1$ & $1,6 \pm 1,8$ & 0,832 & 24,3 & 25,3 & 0,213 \\
\hline $15-19$ & $2,7 \pm 2,3$ & $2,9 \pm 1,9$ & 0,396 & 26,4 & 26,5 & 0,760 \\
\hline $20-24$ & $4,5 \pm 2,4$ & $5,9 \pm 3,3$ & 0,156 & 25,8 & 25,1 & 0,650 \\
\hline $25-34$ & $7,7 \pm 3,8$ & $11,0 \pm 6,3$ & 0,083 & 24,7 & 22,3 & 0,115 \\
\hline $35-44$ & $9,5 \pm 5,1$ & $17,7 \pm 8,0$ & 0,001 & 22,9 & 14,9 & $<0,001$ \\
\hline $45-64$ & $21,4 \pm 2,5$ & $27,8 \pm 5,5$ & 0,212 & 11,4 & 8,9 & 0,374 \\
\hline $65-74$ & $23,5 \pm 7,1$ & $26,3 \pm 7,0$ & 0,233 & 9,8 & 7,1 & 0,282 \\
\hline$\geq 75$ & $28,5 \pm 6,4$ & $28,8 \pm 5,4$ & 0,960 & 4,8 & 3,9 & 0,919 \\
\hline Total & $5,0 \pm 7,9$ & $7,5 \pm 10,3$ & 0,01 & 21,8 & 19,4 & 0,048 \\
\hline
\end{tabular}

* Valor de $\mathrm{p}$ a partir de teste de Mann-Whitney.

cálculo foram estatisticamente maiores no sexo masculino ( $\mathrm{p}=0,017)$. Nas faixas etárias 20 a 24 e 25 a 34 anos a condição periodontal não diferiu entre os sexos. Para a faixa de 35 a 44 anos, enquanto sangramento foi mais comum entre as mulheres $(7,7 \%)$, o cálculo apresentou-se mais freqüente entre os homens $(45,5 \%)(p=0,041)$. A condição periodontal não diferiu entre os sexos a partir dos 45 anos. Na faixa de 45 a 64 anos houve o predomínio de cálculo $(61,1 \%)$, e para as faixas
65 a 74 e 75 ou mais a condição "excluído" foi a mais comum (28\% e $68,5 \%$, respectivamente).

\section{Discussão}

Segundo as diretrizes da Política Nacional de Saúde Bucal 21, a promoção de saúde bucal se integra a uma estratégia mais global de saúde que extrapola a dimensão técnica em odontologia. 
Índice Periodontal Comunitário (CPI) segundo faixa etária e sexo, em indivíduos Guaraní. Rio de Janeiro, Brasil, 2007.

\begin{tabular}{|c|c|c|c|c|c|c|c|c|c|c|c|c|c|c|c|c|}
\hline \multirow{3}{*}{$\begin{array}{l}\text { Faixa etária } \\
\text { (anos) }\end{array}$} & \multicolumn{15}{|c|}{ Condição periodontal mais grave } & \multirow{3}{*}{$\begin{array}{l}\text { Valor } \\
\text { de } p \text { * }\end{array}$} \\
\hline & \multicolumn{3}{|c|}{ Sadio } & \multicolumn{3}{|c|}{ Sangramento } & \multicolumn{3}{|c|}{ Cálculo } & \multicolumn{3}{|c|}{ Bolsa 4-5 } & \multicolumn{3}{|c|}{ Excluído } & \\
\hline & Total & M & $\mathbf{F}$ & Total & M & $\mathbf{F}$ & Total & $\mathbf{M}$ & $F$ & Total & M & $\mathbf{F}$ & Total & M & $\mathrm{F}$ & \\
\hline $\begin{array}{l}12 \\
(n=40)\end{array}$ & 87,5 & 77,8 & 95,5 & 2,8 & 5,6 & 4,5 & 10,0 & 16,7 & 0,0 & 0,0 & 0,0 & 0,0 & 0,0 & 0,0 & 0,0 & 0,220 \\
\hline $\begin{array}{l}15-19 \\
(n=73)\end{array}$ & 68,5 & 51,5 & 82,5 & 19,2 & 30,3 & 10,0 & 12,3 & 18,2 & 7,5 & 0,0 & 0,0 & 0,0 & 0,0 & 0,0 & 0,0 & 0,017 \\
\hline $\begin{array}{l}20-24 \\
(n=29)\end{array}$ & 86,5 & 92,3 & 81,3 & 6,9 & 0,0 & 12,5 & 0,0 & 7,7 & 6,2 & 0,0 & 0,0 & 0,0 & 0,0 & 0,0 & 0,0 & 0,417 \\
\hline $\begin{array}{l}25-34 \\
(n=48)\end{array}$ & 57,1 & 51,7 & 65,0 & 10,2 & 6,9 & 15,0 & 32,7 & 41,4 & 20,0 & 00,0 & 0,0 & 0,0 & 0,0 & 0,0 & 0,0 & 0,249 \\
\hline $\begin{array}{l}35-44 \\
(n=48)\end{array}$ & 62,5 & 45,5 & 76,9 & 6,3 & 4,5 & 7,7 & 27,1 & 45,5 & 11,5 & 2,1 & 4,5 & 0,0 & 2,1 & 0,0 & 3,8 & 0,041 \\
\hline $\begin{array}{l}45-64 \\
(n=18)\end{array}$ & 16,7 & 22,2 & 11,1 & 11,1 & 22,2 & 0,0 & 61,1 & 55,6 & 66,7 & 0,0 & 0,0 & 0,0 & 11,1 & 0,0 & 22,2 & 0,331 \\
\hline $\begin{array}{l}65-74 \\
(n=25)\end{array}$ & 24,0 & 30,8 & 16,7 & 20,0 & 7,7 & 33,3 & 24,0 & 38,5 & 8,3 & 4,0 & 7,7 & 0,0 & 28,0 & 15,4 & 41,7 & 0,138 \\
\hline $\begin{array}{l}\geq 75 \\
(n=19)\end{array}$ & 10,5 & 16,7 & 7,7 & 10,5 & 0,0 & 15,4 & 10,5 & 16,7 & 7,7 & 0,0 & 0,0 & 0,0 & 68,5 & 66,6 & 69,2 & 0,472 \\
\hline Total & 59,6 & 57,7 & 68,0 & 11,3 & 13,1 & 11,0 & 20,9 & 23,8 & 10,5 & 0,70 & 1,2 & 0,0 & 7,6 & 4,2 & 10,5 & 0,002 \\
\hline
\end{tabular}

M: masculino; F: feminino.

* Valor de p a partir de teste do qui-quadrado para comparação entre sexos.

Assim, a saúde bucal se torna parte integrante das demais áreas de saúde coletiva. O uso de estratégias voltadas às comunidades, como oferta de assistência odontológica básica, fluoretação das águas e utilização de creme dental fluoretado, compõe um conjunto de iniciativas fundamentais de uma política de promoção de saúde bucal.

No Brasil, uma criança de 18 a 36 meses já possui, pelo menos, 1 dente atingido por cárie e aos 5 anos esta média aumenta para 2,8 dentes atacados 22. Entre os Guaraní, as médias são ligeiramente menores que os valores nacionais, com 0,7 e 2,6 para as crianças de 18 a 36 meses e 5 anos, respectivamente. Entretanto, quando se analisa a contribuição percentual do componente cariado, os valores nas crianças Guaraní ( $100 \%$ e $90,2 \%)$ ultrapassam os nacionais $(96,3 \%$ e $82,1 \%)$.

No inquérito nacional sobre saúde bucal realizado no Brasil em 2003 (SB Brasil), as crianças com 12 anos e os adolescentes de 15 a 19 apresentaram, respectivamente, uma média de 2,8 e 6,2 dentes atingidos por cárie. Em adultos, o CPOD foi de 20,1 na faixa etária de 35 a 44 anos e 27,8 entre aqueles de 65 a 74 . O componente perdido contribuiu com aproximadamente $66 \%$ do índice no grupo de 35 a 44 anos e com cerca de 93\% no grupo de 65 a 74 22. Entre os Guaraní, a média de elementos dentários permanentes com experiência de cárie aos 12 anos foi de 1,7, valor este inferior à meta da OMS para o ano 2000 23, e entre 15 e 19 anos foi de 2,8. Há de se destacar que, a partir dos 20 anos, a média do CPOD subiu para 5,3. Em adultos Guaraní, o CPOD foi de 13,6 na faixa etária de 35 a 44 anos e 24,8 na de 65 a 74. O componente perdido foi responsável por $78,1 \%$ do índice no grupo de 35 a 44 anos e por $94,3 \%$ no de 65 a 74 . Esses valores demonstram um CPOD aos 12 anos inferior entre os Guaraní quando comparados à média nacional, porém aquém das metas da OMS para o ano 2000. Vale ressaltar, contudo, que nos indivíduos Guaraní a contribuição do componente perdido se manteve em nível superior à média do país, revelando que o tratamento exodôntico (extrações) foi expressivo no passado.

Ainda em relação aos resultados do SB Brasil 22, 40\% das crianças brasileiras de 5 a 6 anos não apresentavam experiência de cárie. Na população Guaraní os achados foram ligeiramente inferiores $(38,5 \%)$, porém também abaixo da meta da OMS para 2000 (50\%) 23. Para as demais faixas etárias, a população Guaraní também apresentou índices melhores do que a população brasileira 22 . 
Os achados deste estudo referentes à proporção de pessoas com 20 ou mais dentes foram semelhantes aos do SB Brasil. Para as faixas etárias 35 a 44 e 65 a 74 anos, as proporções para os Guaraní foram de 54,2\% e 12\%, respectivamente, enquanto no inquérito nacional foram de $54 \%$ e $10,2 \%$. Esses valores, tanto para os Guaraní como para a população brasileira, foram aquém das metas da OMS para 2000 (75\% e 50\%, respectivamente) 23 .

Apesar dos resultados de cárie para os Guaraní serem melhores que as médias nacionais em todas as idades, e a perda dentária em adultos e idosos ter se mostrado semelhante aos achados do inquérito nacional, o nível de saúde bucal dos Guaraní esteve aquém das metas da OMS para 2000, exceto para o CPOD aos 12 anos 23. Os resultados evidenciam que a evolução da cárie desde a infância até os idosos indígenas, bem como a perda dentária, constituem-se em um aspecto relevante no planejamento de medidas preventivas.

Nos últimos anos tem crescido o número de estudos sobre as condições de saúde bucal dos povos indígenas no Brasil. Infelizmente, há dificuldades de comparabilidade entre os resultados devido a diferenças metodológicas, faixas etárias examinadas e ao modelo de assistência odontológica sob consideração, entre outras. No entanto, os resultados da presente investigação podem ser comparados com os achados de Arantes 24, Carneiro 25 e Fratucci 13, que utilizaram metodologias e faixas etárias comparáveis com a presente pesquisa. Esses autores realizaram pesquisas, respectivamente, nas etnias Xavánte (Mato Grosso), Baníwa (Amazonas) e Guaraní (São Paulo).

Em seu estudo sobre os Xavánte, Arantes 24 confirma que o componente obturado pode refletir uma diferenciação no que tange ao acesso aos serviços de atenção odontológica. Enquanto no nível nacional a composição percentual do componente obturado do ceod, aos 5 anos, foi de 12,9\%, entre os Xavánte não foram observados dentes obturados nesta idade índice (ceo-d de 4,9). Para os Guaraní, o componente obturado representou $7,7 \%$ do ceod aos 5 anos, enquanto o componente cariado correspondeu a $92,3 \%$ do valor total do ceod nesta idade. Valores maiores foram encontrados por Carneiro 25 entre os Baníwa do alto Rio Negro (Amazonas), onde se registrou que apenas $1,8 \%$ do ceod $(6,3)$ era de elementos dentários restaurados, aos 5 anos. Também na etnia Guaraní, em estudo realizado em São Paulo, na comunidade de Morro da Saudade, Fratucci 13 observou que, aos 5 anos, os Mbyá tinham um ceod de 3,3, sendo $44 \%$ de crianças livres de cárie, mas com total ausência de elementos restaurados nesta idade.
No que diz respeito às alterações gengivais, apenas o estudo de Fratucci 13 permitiu traçar comparações, demonstrando que aos 5 anos a presença de inflamações gengivais era, aproximadamente, três vezes maior (33,3\%) do que a dos Guaraní pesquisados no Rio de Janeiro (10,2\%).

Como já referido, os Guaraní apresentaram, aos 12 anos, média de CPOD e percentual de dentes cariados inferiores aos valores nacionais 22 . O componente obturado foi responsável por 35,3\% do CPOD aos 12 anos, próximo ao do Brasil (32,7\%). Entre os Xavánte 24, foi de 14,6\%; entre os Guaraní do Morro da Saudade 13, em São Paulo, de 10,9\%; finalmente, entre os Baníwa 25 de 25,2\%. Tanto para os Guaraní do Rio de Janeiro como de São Paulo, os percentuais encontrados revelam uma pior condição de acesso ao tratamento quando comparados aos valores da Região Sudeste derivados do SB Brasil (52,2\%) 22.

No grupo etário de 15 a 19 anos, o CPOD de 2,8 referente aos Guaraní do Rio de Janeiro foi inferior à média nacional $(6,2)$ registrada pelo SB Brasil 22. Um total de 39,9\% dos indivíduos nessa faixa estavam livres de lesões cariosas, enquanto que para o Brasil o valor era de $11,1 \%$. Entre os Xavánte na mesma faixa etária, o CPOD foi de 5,7 e apenas $8 \%$ das pessoas examinadas estavam livres de cárie 24. Entre os Guaraní do Morro da Saudade 13 , o valor do CPOD chega a 5,8 dentes atingidos, com $12,5 \%$ das pessoas livres de cáries. Quanto aos Baníwa, apresentaram um CPOD de 8,2 e $34,9 \%$ das pessoas livres de cárie 25 . Novamente se destaca o valor encontrado entre os Guaraní de São Paulo, onde se observou um percentual de elementos restaurados que demonstra algum acesso ao tratamento odontológico. É também relevante o fato de que nos Baniwa os dentes perdidos superavam em $70 \%$ o valor percentual de elementos restaurados nessa faixa etária, confirmando mais uma vez a provável ausência ou insuficiência de atividades de promoção e prevenção em saúde bucal nesta etnia.

Para o CPI, a OMS indica a idade de 15 a 19 anos como uma das faixas etárias de referência. Os jovens Xavánte 24 nessa faixa etária apresentaram $44,7 \%$ dos sextantes com sangramento, $37,6 \%$ com cálculo e $0 \%$ com bolsas periodontais de 4 a $5 \mathrm{~mm}$. Nos Guaraní de São Paulo 13, as condições eram piores, respectivamente de $60 \%$, $20 \%$ e $20 \%$. No caso dos Guaraní investigados neste estudo, 19,2\% dos sextantes dos indivíduos na faixa etária apresentaram sangramento, 12,3\% cálculo e não foram identificados sextantes com bolsas de 4 a $5 \mathrm{~mm}$. Portanto, do ponto de vista comparativo, os Guaraní do Rio de Janeiro apresentam melhor condição periodontal do que a reportada em outros estudos sobre povos indígenas no país. 
Entre os Guaraní investigados na faixa etária de 20 a 24 anos, a porcentagem de elementos obturados alcançou seu maior valor $(37,7 \%)$, superando os componentes cariado e perdido. Entretanto, nessa faixa observou-se um valor percentual de elementos perdidos cerca de três vezes maior $(32,1 \%)$ do que o encontrado aos 12 anos (11,8\%). Entre os Xavánte 24 , o percentual de elementos perdidos $(39,4 \%)$ superou em cerca de três vezes o de obturados (13\%). Entre os Baníwa 25, o percentual de dentes perdidos $(65,9 \%)$ supera em mais de dez vezes aquele do componente obturado $(5,8 \%)$.

Os adultos Guaraní entre 35 e 44 anos apresentaram uma média de 13,9 dentes atingidos por cárie, enquanto a média nacional foi de 20,1 22. O componente perdido foi também mais elevado nesse grupo etário, representando $76,2 \%$ do CPOD, com impacto muito maior entre as mulheres $(\mathrm{CPOD}=17,7)$ do que nos homens $(\mathrm{CPOD}=9,5)$. Entre os Xavánte, a diferença entre sexos no grupo etário com mais de 35 anos também foi observada, com CPOD de 14,9 para homens e 20,2 para mulheres 24. Em relação ao estudo de Carneiro 25, os valores de CPOD encontrados entre 40 e 49 anos foram de 21,4 para as mulheres e 13,7 para os homens.

Com base nas comparações dos resultados sobre o perfil de saúde bucal dos Guaraní do Rio de Janeiro com os das três outras populações indígenas, evidencia-se um melhor grau de acesso às ações e serviços de saúde na etnia Guaraní do Rio de Janeiro. Há de se destacar que as aldeias Guaraní adscritas ao Pólo-Base de Angra dos Reis têm uma população menor (617 indivíduos) e menos dispersa em relação aos Xavánte 24 e aos Baníwa 24. É possível que a maior facilidade de acesso no caso Guaraní possibilite o desenvolvimento de atividades ligadas à promoção de saúde e à atenção odontológica propriamente dita. Vale ressaltar que, além disso, vem sendo provida assistência à saúde bucal aos Guaraní do Rio de Janeiro, de forma continuada, desde 1993 12,18, o que não encontra paralelo nos demais estudos de caso. Apesar do estudo de Fratucci 13 também abordar a etnia Guaraní, em São Paulo, deve-se destacar que a assistência odontológica oferecida na época (em 2000) ainda tinha um caráter incipiente.

A partir de inquéritos transversais realizados entre os Xavánte, Arantes 24 observou valores de CPOD mais elevados em mulheres, assim como maiores níveis de perdas dentárias. Buscando aprofundar as análises, Arantes conduziu um estudo prospectivo, no qual observou que as mulheres entre 20 e 34 anos apresentaram maiores incidências de cárie que os homens. Segundo o autor, essa diferença pode ser explicada por fa- tores ligados a questões de gênero. No que diz respeito à inserção das mulheres na economia Xavánte, são elas que preparam e cuidam dos alimentos consumidos pela família. Arantes argumenta que, nos dias atuais, há hábitos alimentares diferenciados entre os sexos, com uma maior exposição das mulheres ao consumo de alimentos industrializados e ricos em carboidratos 24,26 . Observa-se também uma elevada taxa de fecundidade total, da ordem de oito filhos por mulher durante o período reprodutivo 24 . É também prolongado o período de amamentação na sociedade Xavánte. Há indicações de que as transformações hormonais e metabólicas decorrentes da gestação e lactação podem estimular alterações no meio bucal. Alguns estudos sugerem que a elevação do nível de estrógeno na saliva provocaria descamação do tecido epitelial, além de redução do pH e da capacidade tampão da saliva, originando um ambiente favorável à reprodução bacteriana. Dessa forma, Streptococcus mutans e lactobacilos poderiam se multiplicar com mais facilidade. Os níveis salivares de fosfato e cálcio também se reduziriam no período gestacional, propiciando simultaneamente a diminuição da capacidade de remineralização, pela saliva, das lesões primárias da cárie. Dessa maneira, a gestação tornaria o meio bucal mais apropriado ao desenvolvimento de cárie e periodontopatias 27 .

Os resultados para os Guaraní do Rio de Janeiro, que se baseiam em dados transversais, evidenciam achados semelhantes àqueles dos Xavánte, no sentido de valores de CPOD e de perda dentária mais expressivos nas mulheres. Ainda que questões ligadas à alimentação, estilo de vida e reprodução possam também estar relacionadas aos diferenciais de saúde bucal entre homens e mulheres Guaraní, são necessárias mais investigações com vistas a compreender tais diferenças.

No que se refere à coleta e processamento de dados, o SIASI, criado pela FUNASA em 2000, tem por perspectiva implementar um módulo de saúde bucal. Tal módulo, cuja implantação já deveria ter ocorrido, tem como objetivo, uma vez em operação, sistematizar e disponibilizar as informações necessárias para um monitoramento das condições de saúde bucal ${ }^{28}$. No caso dos Guaraní, assim como no das demais etnias que estão experimentando importantes modificações nos determinantes de saúde bucal, um monitoramento contínuo pode assumir particular importância. O atual quadro epidemiológico de saúde bucal Guaraní, que se insere em um contexto mais amplo da realidade indígena no país, também revela a necessidade de se conceber modelos de atenção mais específicos para os povos indígenas 29 . 


\section{Resumo}

Este estudo teve por objetivo descrever a situação de saúde bucal em índios Guaraní do Estado do Rio de Janeiro, Brasil. Um inquérito epidemiológico em saúde bucal, utilizando critérios da Organização Mundial da Saúde foi realizado em 508 sujeitos (90,7\% da população). Aos 5 anos, 38,5\% das crianças estavam livres de cárie e aos 12 o CPOD foi igual a 1,7. Observou-se que $54,2 \%$ dos indivíduos entre 35-44 anos e $12 \%$ entre 65-74 apresentavam 20 ou mais dentes. $O$ número de dentes para 35-44 e 65-74 anos foi de 18,5 $e$ 8,5. Enquanto o ceod não diferiu entre sexos, o CPOD e a perda dentária foram maiores em mulheres. Aproximadamente $60 \%$ da população não demonstraram problemas periodontais, enquanto 20,9\% e 11,3\% apresentaram sangramento gengival e cálculo como condições periodontais mais graves. Sangramento e cálculo foram mais comuns em homens, e sextantes excluídos em mulheres. Ações de promoção de saúde bucal são necessárias para esse grupo étnico e devem contemplar especificidades para diferentes grupos etários e de gênero.

Cárie Dentária; Saúde Bucal; Índios Sul-Americanos

\section{Referências}

1. Arantes R. Saúde oral de uma comunidade indígena Xavante do Brasil Central: uma abordagem epidemiológica e bioantropológica [Dissertação de Mestrado]. Rio de Janeiro: Escola Nacional de Saúde Pública, Fundação Oswaldo Cruz; 1998.

2. Arantes R, Santos RV, Coimbra Jr. CEA. Saúde bucal na população indígena Xavánte em Pimentel Barbosa, Mato Grosso, Brasil. Cad Saúde Pública 2001; 17:375-84.

3. Pinto VG. Saúde bucal coletiva. São Paulo: Livraria Santos Editora; 2000.

4. Fundação Nacional de Saúde. Diretrizes para a atenção à saúde bucal nos Distritos Sanitários Especiais Indígenas: manual técnico. Brasília: Fundação Nacional de Saúde; 2007.

5. Arantes R. Saúde bucal dos povos indígenas no Brasil: panorama atual e perspectivas. In: Coimbra Jr. CEA, Santos RV, Escobar AL, organizadores. Epidemiologia e saúde dos povos indígenas do Brasil. Rio de Janeiro: Editora Fiocruz/ABRASCO 2003: p. 49-72.

6. Pose SB. Avaliação das condições de saúde bucal dos índios Xavante do Brasil Central [Dissertação de Mestrado]. Rio de Janeiro: Escola Nacional de Saúde Pública, Fundação Oswaldo Cruz; 1993.

\section{Colaboradores}

P. Alves Filho participou do planejamento do estudo, realizou a coleta e análise dos dados e redigiu o artigo. R.V. Santos participou da elaboração do estudo, contribuiu na análise e redação do texto e realizou a revisão final do artigo. M.V. Vettore participou da análise dos dados e contribuiu na redação e na revisão final do artigo.

\section{Agradecimentos}

Nossos agradecimentos aos Guaraní de Sapukai, Itaxĩ, Guyraitapú, Arandumirĩ e Jahapé. Este trabalho não seria possível sem a anuência dessas comunidades. Nossos agradecimentos, também, à FUNASA e à Função Nacional do Índio (FUNAI) pelo apoio durante a realização do estudo de mestrado (P.A.F.) que originou este artigo.
7. Coimbra Jr. CEA, Flowers NM, Salzano FM, Santos RV. The Xavante in transition: health, ecology and bioanthropology in Central Brazil. Ann Arbor: University of Michigan Press; 2002.

8. Parizotto SPCOL. Prevalência de cárie dentária na dentição decídua de crianças da comunidade indígena Kaiowá-Guaraní de Mato Grosso do Sul e associação com fatores de risco [Tese de Doutorado]. São Paulo: Faculdade de Odontologia, Universidade de São Paulo; 2004.

9. Detogni A. De volta às origens. Rev ABO Nac 1994; 2:138-48.

10. Rigonatto DL, Antunes JL, Frazão P. Dental caries experience in Indians of the upper Xingu, Brasil. Rev Inst Med Trop São Paulo 2001; 43:93-8.

11. Tricerri FJ. Breve passagem odontológica entre os índios do Alto Solimões, Amazonas, Brasil. Rev Fund SESP 1985; 30:151-60.

12. Alves Filho P, Ribeiro NBC. Atenção à saúde bucal no programa de saúde indígena do Rio de Janeiro. In: Associação Brasileira de Odontologia do Rio de Janeiro, organizador. Novos rumos da saúde bucal: os caminhos da integralidade. Rio de Janeiro: Associação Brasileira de Odontologia do Rio de Janeiro; 2005. p. 75-87. 
13. Fratucci MVB. Alguns aspectos das condições de Saúde Bucal de uma população indígena Guaraní M’bya no Município de São Paulo [Dissertação de Mestrado]. São Paulo: Faculdade de Saúde Pública, Universidade de São Paulo; 2000.

14. Farias LS, Verani CBL. Componente antropológico. In: Confalonieri UEC, Marinho DP, organizadores. Projeto de antropologia e saúde para a etnia Guaraní de Paraty, Rio de Janeiro: relatório final. Rio de Janeiro: Núcleo de Estudos em Populações Indígenas, Fundação Oswaldo Cruz; 1998.

15. Diretoria de Assuntos Fundiários, Fundação Nacional do Índio. Memorial descritivo de demarcação. Brasília: Fundação Nacional do Índio; 1991.

16. Diretoria de Assuntos Fundiários, Fundação Nacional do Índio. Memorial descritivo de demarcação - 16 de janeiro. Brasília: Fundação Nacional do Índio; 1995.

17. Diretoria de Assuntos Fundiários, Fundação Nacional do Índio. Memorial descritivo de demarcação - 25 de agosto. Brasília: Fundação Nacional do Índio; 1995.

18. Alves Filho P. A saúde bucal dos índios Guaraní no Estado do Rio de Janeiro [Dissertação de Mestrado]. Rio de Janeiro: Escola Nacional de Saúde Pública, Fundação Oswaldo Cruz; 2007.

19. Área Técnica de Saúde Bucal, Departamento de Atenção Básica, Secretaria de Políticas de Saúde, Ministério da Saúde. Projeto SB2000: condições de saúde bucal da população brasileira no ano 2000 : manual do examinador. Brasília: Ministério da Saúde; 2001.

20. World Health Organization. Oral health surveys - basic methods 4. Geneva: World Health Organization; 1997.
21. Coordenação Nacional de Saúde Bucal, Departamento de Atenção Básica, Secretaria de Atenção à Saúde, Ministério da Saúde. Política Nacional de Saúde Bucal. Brasília: Ministério da Saúde; 2004.

22. Ministério da Saúde. Projeto SB Brasil 2003: condições de saúde bucal da população brasileira 2002-2003: resultados principais. Brasília: Ministério da Saúde; 2004.

23. Fédération Dentaire Internationale. Global goals for oral health in the year 2000. Int Dent J 1982; 32:74-7.

24. Arantes R. Saúde bucal dos povos indígenas no Brasil e o caso dos Xavante de Mato Grosso [Tese de Doutorado]. Rio de Janeiro: Escola Nacional de Saúde Pública, Fundação Oswaldo Cruz; 2005.

25. Carneiro MCG. Um olhar sobre os índios Baniwa: saúde bucal e atenção odontológica na região do Alto Rio Negro, Amazônia brasileira, 2000 a 2004 [Dissertação de Mestrado]. Rio de Janeiro: Escola Nacional de Saúde Pública, Fundação Oswaldo Cruz; 2005.

26. Giaccaria B, Heide A. Xavante, povo autêntico. São Paulo: Editora Salesiana Dom Bosco; 1984.

27. Laine MA. Effect of pregnancy on periodontal and dental health. Acta Odontol Scand 2002; 60:257-64.

28. Sousa MC, Scatena JHG, Santos RV. O Sistema de Informação da Atenção à Saúde Indígena (SIASI): criação, estrutura e funcionamento. Cad Saúde Pública 2007; 23:853-61.

29. Paim JS. Modelos de atenção e vigilância em saúde In: Rouquayrol MZ, organizador. Epidemiologia \& saúde. Rio de Janeiro: MEDSI; 2004. p. 567-86.

Recebido em 07/Abr/2008

Versão final reapresentada em 11/Jun/2008

Aprovado em 12/Jun/2008 